\title{
Autoimmunity and long-term safety and efficacy of alemtuzumab for multiple sclerosis: Benefit/risk following review of trial and post-marketing data
}

\author{
Alasdair J Coles*, Joanne L Jones*, Patrick Vermersch, Anthony Traboulsee, \\ Ann D Bass, Aaron Boster iD, Andrew Chan, Giancarlo Comi, Óscar Fernández, \\ Gavin Giovannoni, Eva Kubala Havrdova, Christopher LaGanke, Xavier Montalban, \\ Celia Oreja-Guevara iD, Fredrik Piehl, Heinz Wiendl iD and Tjalf Ziemssen iD on \\ behalf of the CAMMS223, CARE-MS I, CARE-MS II, CAMMS03409, and TOPAZ investigators
}

Abstract: Does preexisting or treatment-emergent autoimmunity increase the risk of subsequent autoimmune disease in individuals with relapsing-remitting multiple sclerosis (MS) after alemtuzumab? In the extended phase 2/3 trials, 34/96 (35.4\%) patients with and 395/1120 (35.3\%) without preexisting autoimmunity developed non-MS autoimmunity. Thyroid autoimmunity after alemtuzumab courses 1 or 2 did not increase subsequent non-thyroid autoimmune adverse events. Therefore, autoimmune disease before or after alemtuzumab treatment does not predict autoimmunity after further courses, so should not preclude adequate alemtuzumab dosing to control MS. Finally, post-marketing safety data contribute toward a full record of the alemtuzumab benefit/risk profile for the MS field.

Keywords: Multiple sclerosis, alemtuzumab, autoimmunity, treatment outcome, risk assessment, product surveillance, post-marketing

Date received: 25 June 2021; revised: 28 September 2021; accepted: 18 October 2021

\section{Introduction}

In 2019, the European Medicines Agency altered the license of alemtuzumab, a high-efficacy drug for multiple sclerosis (MS), to contraindicate use in patients with "concomitant autoimmune diseases (besides MS)," which we interpret as preexisting autoimmunity or autoimmunity emerging after alemtuzumab. ${ }^{1}$ This change was designed to reduce risk of rare, serious events, such as immune thrombocytopenia or autoimmune renal or liver disease. Other rare but serious side effects were also considered, including peri-infusion cardiovascular adverse events (AEs). ${ }^{1}$

We report data from clinical trials on risk of subsequent autoimmune AEs in alemtuzumab-treated patients with preexisting or treatment-emergent autoimmunity. We additionally report safety events encountered post-marketing to contribute toward a complete overview of risks of alemtuzumab.

\section{Methods}

Designs of the CAMMS223 (NCT00050778) and CARE-MS studies (NCT00530348, NCT00548405) and extensions (NCT00930553 and NCT02255656) were described previously. ${ }^{2-6}$ Exclusion criteria included antithyroid stimulating hormone receptor antibodies or clinically significant autoimmunity, including immune cytopenias, rheumatoid arthritis, systemic lupus erythematosus, other connective tissue disorders, vasculitis, inflammatory bowel disease, and severe psoriasis.

Clinical trial data were assessed for impact of preexisting non-MS autoimmunity on risk of developing autoimmunity after alemtuzumab. We also assessed whether emergence of the commonest autoimmune disease after alemtuzumab, thyroid disease, after courses 1 and 2 was associated with development of autoimmune nephropathy, immune thrombocytopenia, acquired hemophilia, or antiglomerular basement membrane disease following
Multiple Sclerosis Journal

$1-5$

DOI: $10.1177 /$ 13524585211061335

(C) The Author(s), 2021 (c) (i) \$

Article reuse guidelines: sagepub.com/journalspermissions
Correspondence to: AJ Coles Department of Clinical Neuroscience, School of Medicine, University of Cambridge, Box 165, Level 5 Block A, Addenbrooke's Hospital, Hills Road, Cambridge CB2 0QQ, UK. ajc1020@medschl.cam. ac.uk

Alasdair J Coles Joanne L Jones Department of Clinical Neuroscience, School of Medicine, University of Cambridge, Cambridge, UK Patrick Vermersch Univ. Lille, INSERM UMR-S1172-Lille Neuroscience et Cognition, CHU Lille, FHU Precise, Lille, France

Anthony Traboulsee The University of British Columbia, Vancouver, BC, Canada

Ann D Bass

Neurology Center of San Antonio, San Antonio, TX, USA

Aaron Boster Boster MS Center, Columbus, OH, USA Andrew Chan Bern University Hospital and University of Bern, Bern, Switzerland

Giancarlo Comi Università Vita Salute San Raffaele, Milan, Italy

Óscar Fernández Fundacion IMABIS, Hospital Universitario Carlos Haya, Málaga, Spain

Gavin Giovannoni Barts and The London School of Medicine, Queen Mary University of London, London, UK 
Eva Kubala Havrdova Department of Neurology, First Medical Faculty, Charles University, Prague, Czech Republic

Christopher LaGanke North Central Neurology Associates, Cullman, AL, USA

Xavier Montalban

Department of NeurologyNeuroimmunology, Centre d'Esclerosi Múltiple de Catalunya (Cemcat), Hospital Universitari Vall d'Hebron, Barcelona, Spain

Celia Oreja-Guevara Department of Neurology, Hospital Clínico San Carlos, IdISSC, Madrid, Spain/ Departamento de Medicina, Facultad de Medicina, Universidad Complutense de Madrid (UCM), Madrid, Spain

Fredrik Piehl

Neuroimmunology Unit, Department of Clinical Neuroscience, Karolinska Institutet, Stockholm,

Sweden

Heinz Wiendl University of Münster, Münster, Germany

Tjalf Ziemssen

Center of Clinical Neuroscience, Department of Neurology Carl Gustav Carus University Hospital, Dresden University of Technology, Dresden, Germany

${ }^{*}$ Co-first authors.

Table 1. Effect of preexisting non-MS autoimmunity on the development of additional autoimmunities in clinical trials.

\begin{tabular}{lrlrl}
\hline Preexisting autoimmunity & $N$ & $\begin{array}{l}\text { No post-baseline } \\
\text { autoimmune AE, } \\
n(\%)\end{array}$ & $\begin{array}{l}\text { Exactly 1 post- } \\
\text { baseline autoimmune } \\
\text { AE, } n(\%)\end{array}$ & $\begin{array}{l}\text { 2 } 2 \text { post-baseline } \\
\text { autoimmune AE, } \\
n(\%)\end{array}$ \\
\hline Patients with no preexisting autoimmunity & 1120 & $725(64.7)$ & $303(27.1)$ & $92(8.2)$ \\
Patients with preexisting autoimmunity & 96 & $62(64.6)$ & $29(30.2)$ & $5(5.2)$ \\
Hypothyroidism & 37 & $26(70.3)$ & $10(27.0)$ & $1(2.7)$ \\
Hyperthyroidism & 7 & $4(57.1)$ & $3(42.9)$ & 0 \\
Autoimmune thyroiditis & 6 & $4(66.7)$ & 0 & 0 \\
Eczema & 19 & $7(36.8)$ & $12(63.2)$ & 0 \\
Psoriasis & 19 & $15(79.0)$ & $4(21.1)$ & $1(7.1)$ \\
Alopecia & 14 & $9(64.3)$ & $4(28.6)$ & $1(100)$ \\
Crohn's disease & 1 & 0 & 0 & 0
\end{tabular}

AE: adverse event; MS: multiple sclerosis; SC IFNB-1a: subcutaneous interferon beta-1a.

Pooled CAMMS223, CARE-MS I, and CARE-MS II trials for patients treated with alemtuzumab $12 \mathrm{mg} /$ day or patients treated with SC IFNB-1a in the core studies who switched to alemtuzumab $12 \mathrm{mg} /$ day in the extension studies. Preexisting autoimmune events included the following: hypothyroidism, hyperthyroidism, autoimmune thyroiditis, psoriasis, eczema, alopecia, antiphospholipid syndrome, Crohn's disease, and Guillain-Barré syndrome. Post-baseline autoimmune events included the following: hypothyroidism, hyperthyroidism, autoimmune thyroiditis, psoriasis, eczema, alopecia, antiphospholipid syndrome, Crohn's disease, Guillain-Barré syndrome, acquired hemophilia, antiglomerular basement membrane antibody positive, nephropathy, and immune thrombocytopenia. Serious post-baseline autoimmune AEs occurred in one patient with preexisting hypothyroidism, one patient with preexisting psoriasis, and one patient with preexisting Crohn's disease. An event was considered at post-baseline if it occurred after the first alemtuzumab dose up to 9 years (for patients treated with alemtuzumab $12 \mathrm{mg} /$ day), and if it occurred within 7 years from first alemtuzumab dose (for patients treated with SC IFNB-1a in the core studies and who switched to alemtuzumab $12 \mathrm{mg}$ /day in the extension studies)

subsequent treatment courses. Statistics were performed via chi-square tests. Rates of malignancies, serious infections, Listeria infections, herpetic infections, and deaths were also reported.

Novel AEs reported post-marketing were assessed from the manufacturer's pharmacovigilance database and divided into peri-infusion (during or within 7 days post-infusion) or delayed events (peak incidence 1836 months post-treatment).

Autoimmune AE monitoring was conducted per protocol in clinical trials ${ }^{2,4,5}$ and per labeling and the Risk Evaluation and Mitigation Strategy/Risk Management Plan post-marketing. ${ }^{7}$ The risk of delayed autoimmune events was defined as peak incidence occurring 18-36 months post-treatment.

\section{Results}

\section{Clinical trial efficacy and patient retention}

Efficacy of alemtuzumab versus subcutaneous interferon beta-1a was established in the core clinical studies ${ }^{2,4,5}$ and was maintained long term. Over 9years in pooled CARE-MS I and II alemtuzumab patients, $64 \%$ remained free of 6-month confirmed disability worsening, 45\% achieved 6-month confirmed disability improvement, mean Expanded Disability Status Scale score change was +0.21 , and cumulative median brain volume change was $-1.65 \% .^{6}$ Patient retention rates were 75\% (CARE-MS I) and 66\% (CARE-MS II) through year 9.6

\section{Clinical trial safety outcomes}

Autoimmune AEs after alemtuzumab were similar between patients with preexisting non-MS autoimmunity (34/96 patients $(35.4 \%)$ and those without (395/1120 $(35.3 \%)$, Table 1). Preexisting autoimmunity did not predict additional autoimmunity (Table 1). In patients with or without preexisting autoimmunity, post-alemtuzumab autoimmune AEs were serious in 8.8\% (3/34) and $9.1 \%(36 / 395)$ of cases, respectively. In CAMMS223, $66.7 \%$ of the $9 / 119$ patients with baseline antithyroperoxidase (anti-TPO) antibodies developed autoimmunity after alemtuzumab versus $26.4 \%$ of $110 / 119$ patients without these antibodies $(p=0.0107)$. Of CARE-MS patients with high $(>34 \mathrm{IU} / \mathrm{mL}, n=80 / 1084)$ or normal (1-34IU/mL, $n=1004 / 1084)$ baseline anti-TPO antibody levels, $51.3 \%$ and $34.6 \%$ developed post-baseline autoimmunity, respectively ( $p=0.0027$ ).

Autoimmune AE incidence after course 2 was similar and low in patients with or without thyroid AEs between courses 1 and $2(0 / 53(0 \%)$ vs 25/841 (3.0\%), 


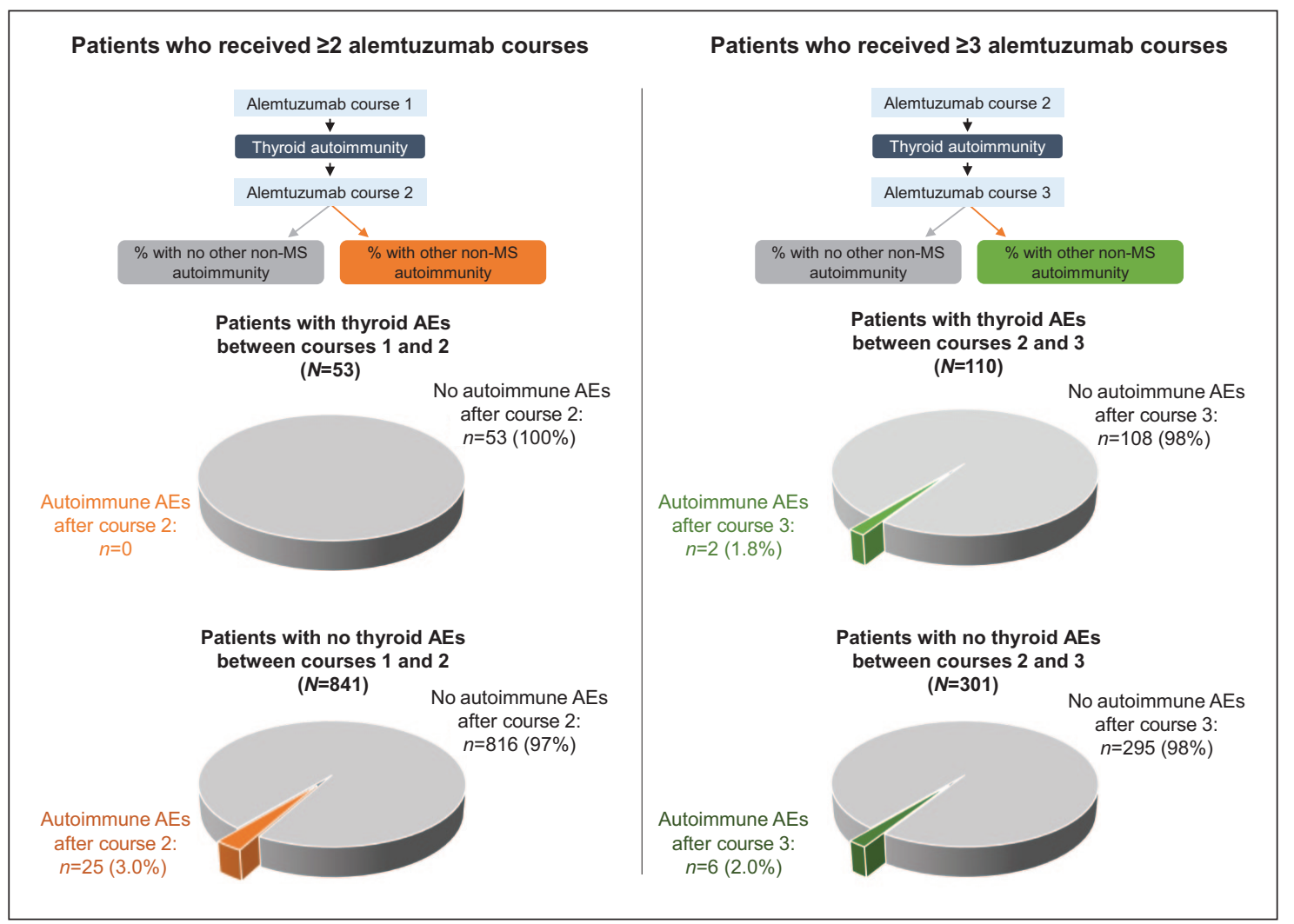

Figure 1. Effect of post-alemtuzumab thyroid AEs on subsequent development of immune thrombocytopenia, acquired hemophilia, antiglomerular basement membrane antibody positive, or nephropathy in clinical trials.

AE: adverse event; MS: multiple sclerosis.

Pooled CAMMS223, CARE-MS I, and CARE-MS II trials for patients treated with alemtuzumab $12 \mathrm{mg} /$ day. Treatment-emergent thyroid AEs include the following: antithyroid antibody positive, autoimmune thyroiditis, Basedow's disease, blood thyroid stimulating hormone abnormal, blood thyroid stimulating hormone decreased, blood thyroid stimulating hormone increased, endocrine ophthalmopathy, exophthalmos, goiter, hyperthyroidism, hypothyroidic goiter, hypothyroidism, myxedema, primary hypothyroidism, thyroid cyst, thyroid dermatopathy, thyroid disorder, thyroid function test abnormal, thyroid mass, thyroid pain, thyroidectomy, thyroiditis, thyroiditis subacute, thyrotoxicosis, thyroxine binding globulin increased, thyroxine decreased, thyroxine free decreased, thyroxine free increased, thyroxine increased, toxic nodular goiter, triiodothyronine decreased, triiodothyronine free decreased, triiodothyronine free increased, triiodothyronine increased, and triiodothyronine uptake decreased. Subsequent autoimmune events include any nephropathy event, any immune thrombocytopenia event, acquired hemophilia, and antiglomerular basement membrane antibody positive.

Figure 1). Likewise, autoimmune AE rates after course 3 were similar and low between patients with and without thyroid AEs between courses 2 and $3(2 / 110$ $(1.8 \%)$ vs $6 / 301(2.0 \%)$.

In patients treated with alemtuzumab $12 \mathrm{mg}(N=1216$ 9021 patient-years' follow-up), 26 (2.1\%) experienced malignancies; this unadjusted incidence rate is not higher than the age-adjusted rate in the overall US population (2.88 vs 4.42 per 1000 person-years, respectively). ${ }^{8}$ Serious post-alemtuzumab infections occurred in 113 patients (9.3\%). Listeria meningitis (grade 2) occurred in one patient, 12 days after completing course 1 of alemtuzumab $24 \mathrm{mg}$. Herpetic infections occurred in 330 patients $(27.1 \%)$, most commonly herpes zoster $(n=173(14.2 \%)$ and oral herpes $(n=130(10.7 \%))$. Nine $(0.74 \%)$ and two $(0.16 \%)$ patients experienced myocardial infarctions or stroke (ischemic stroke and cerebral hemorrhage), respectively, occurring $\geqslant 136$ days postinfusion. Over 9years, $19(1.6 \%)$ deaths (2.1 per 1000 patient-years) were reported in patients exposed to alemtuzumab $12 \mathrm{mg}$ (overall age-adjusted US death rate: 7.3 per 1000 person-years). ${ }^{9}$

\section{Post-marketing safety}

As of 31 March 2019, post-marketing data included 25,292 patients with alemtuzumab exposure.

Peri-infusion AEs reported post-marketing included hemorrhagic stroke (2.8 in 10,000 patients), pulmonary alveolar hemorrhage (4.3 in 10,000 (unrelated to antiglomerular basement membrane disease)), other stroke (0.8 in 10,000), myocardial infarction (2.0 in 10,000), and cervicocephalic arterial dissection (1.6 in 10,000). Among 21 cases of symptoms/signs consistent with 
acute coronary syndrome within 72 hours post-infusion, none were fatal, three had acute myocardial infarction, and nine were in $\leqslant 40$-year-old patients. A majority $(15 / 21)$ had risk factors for cardiovascular disease. Of the 13 patients with hemorrhagic or ischemic stroke, seven reported "blood pressure increase" during infusion. Ischemic strokes were associated with cervicocephalic arterial dissection. One hemorrhagic stroke was fatal.

The reported post-marketing cases of delayed autoimmune hepatitis $(10.7$ in 10,000$)$ had 12 - to 29 -month onset time. No autoimmune hepatitis cases occurred $>4$ years after dosing. Reported hemophagocytic lymphohistiocytosis cases $(2.7$ in 10,000$)$ occurred a few months to 4 years following treatment initiation, with two associated deaths.

Listeriosis was reported in 77 patients post-marketing (30.4 in 10,000).

\section{Discussion}

In this analysis of clinical trials, preexisting autoimmunity did not predict autoimmunity emerging after alemtuzumab. This conclusion is based on 96 patients with baseline autoimmunity because serious autoimmune disease was an exclusion criterion. Likewise, from the larger cohort without preexisting autoimmunity $(N=1120)$, emergence of thyroid autoimmunity after the first or second course, usually in the third year following treatment, ${ }^{2-6}$ did not increase the risk of further autoimmunity after subsequent courses. Importantly, we did observe significantly higher risk of post-alemtuzumab autoimmunity in patients with positive or higher than normal anti-TPO antibodies. Post-marketing data confirmed low absolute risk for peri-infusion, non-autoimmune AEs, including cardiovascular and pulmonary events, consistent with product labeling.

In our opinion, these findings, coupled with knowledge that thyroid disorders with alemtuzumab are rarely serious, ${ }^{10}$ suggest thyroid autoimmunity should not preclude further alemtuzumab treatment. Instead, this contraindication leaves patients - who would otherwise be appropriate candidates - incompletely treated and denied the full benefit of alemtuzumab.

\section{Acknowledgements}

The authors and Sanofi thank the patients for their participation in the trials, as well as the CAMMS223 and CARE-MS Steering Committees and CAMMS03409 and TOPAZ Investigators. Critical review of the manuscript was provided by Alana T Wong, $\mathrm{PhD}$, of Sanofi. Statistical support was provided by Elizabeth M Poole,
$\mathrm{PhD}$, an employee of Sanofi when this study was undertaken (current affiliation: bluebird bio). Editorial and writing assistance was provided by Richard J Hogan, $\mathrm{PhD}$, and Valerie P Zediak, PhD, of Eloquent Scientific Solutions, a division of Envision Pharma Group.

\section{Declaration of Conflicting Interests}

The author(s) declared the following potential conflicts of interest with respect to the research, authorship, and/ or publication of this article: A.J.C. received consulting fees, lecture fees, and institutional grant support from Sanofi up to September 2017. J.L.J. received consulting fees and grant support (Sanofi). P.V. received consulting and/or speaking fees and research support (Almirall, Biogen, Celgene, Merck Serono, Novartis, Roche, Sanofi, Servier, and Teva). A.T. received consulting and/ or speaking fees and grant/research support (Biogen, Chugai, Roche, Sanofi, and Teva). A.D.B. received consulting fees/fees for non-CME services from commercial interests or their agents/grant and research support (Biogen, EMD Serono, Mallinckrodt, Novartis, RocheGenentech, Sanofi, and TG Therapeutics). A.B. received consulting fees and/or fees for non-CME services (Biogen, Mallinckrodt, Medtronic, Novartis, Sanofi, and Teva). A.C. received honoraria for presentations or advisory boards to support university/hospital research funds (Actelion, Almirall, Bayer, Biogen, Celgene, Merck, Novartis, Roche, Sanofi, and Teva) and research support (Biogen, Sanofi, and UCB). G.C. received consulting fees (Actelion, Bayer Schering, Merck Serono, Novartis, Sanofi, and Teva) and lecture fees (Bayer Schering, Biogen Dompé, Merck Serono, Novartis, Sanofi, Serono, Symposia International Foundation, and Teva). Ó.F. received speaking and/or consulting fees (Allergan, Almirall, Bayer Schering, Biogen, Merck Serono, Novartis, Sanofi, and Teva); compensation for serving as a journal editor, associate editor, or member of an editorial advisory board (Revista Española de Esclerosis Múltiple); and research support (Hospital Foundation FIMABIS). G.G. received consulting and/or grant/research support (AbbVie, Actelion, Atara Biotherapeutics, Bayer, Biogen, Canbex Therapeutics, Five Prime Therapeutics, GSK, GW Pharma, Merck, Merck Serono, Novartis, Oxford PharmaGenesis, Protein Discovery Laboratories, Roche, Sanofi Genzyme, Synthon, Teva, and UCB). E.K.H. received honoraria and grant support (Actelion, Biogen, Merck Serono, Novartis, Receptos, Roche, Sanofi, and Teva), and support (Ministry of Education of Czech Republic (PROGRES Q27/LF1)). C.L. received compensation for consulting (Acorda Therapeutics, Bayer, Biogen, Cephalon, EMD Serono, Novartis, Pfizer, Questcor, Sanofi, Strativa, Teva, and UCB). X.M. received speaking honoraria and travel expenses for scientific meetings, steering committee member of clinical trials or 
participated in advisory boards of clinical trials in the past 3 years (Actelion, Alexion, Bayer, Biogen, Celgene, EMD Serono, EXCEMED, Genzyme, MedDay, Merck, MSIF, NervGen, NMSS, Novartis, Roche, Sanofi Genzyme, Teva Pharmaceutical, and TG Therapeutics). C.O.-G. received speaking and/or consultancy fees (Bayer, Biogen, Merck Serono, Novartis, Roche, Sanofi, and Teva). F.P. received research grants (Genzyme, Merck KGaA, and Novartis) and fees for clinical trials (serving as chair of data monitoring committee with Parexel). H.W. received consulting and/or speaking fees (Bayer, Behring, Biogen, EMD Serono, Fresenius Medical Care, Merck Serono, Novartis, Roche, Sanofi, and Teva), license fee payments (Huber Verlag), and grant/research support (Neotope Biosciences, Novartis, and PML Consortium). T.Z. received consulting and/or speaking fees (Alexion, Almirall, Biogen, Celgene, Merck, Novartis, Roche, Sanofi, and Teva) and grant/ research support (Biogen, Novartis, Roche, Sanofi, and Teva).

\section{Funding}

The author(s) disclosed receipt of the following financial support for the research, authorship, and /or publication of this article: The CAMMS223 and CARE-MS studies, and their extensions, were supported by Sanofi and Bayer Healthcare Pharmaceuticals. Editorial and writing assistance was supported by Sanofi.

\section{Previous Presentation of Data}

Some of the data included in this manuscript were presented at the 6th Congress of the European Academy of Neurology, 23-26 May 2020 (Virtual Congress), and the Virtual Joint ACTRIMS-ECTRIMS 2020 Meeting, 11-13 September (MSVirtual2020).

\section{Research Ethics and Patient Consent}

This study was conducted in accordance with the ethical principles outlined in the Declaration of Helsinki. Patients enrolled in the CAMMS223, CARE-MS I, CARE-MS II, CAMMS03409, and TOPAZ studies provided written informed consent, and all procedures were approved by local institutional ethics review boards of participating sites.

\section{ORCID iDs}

Aaron Boster (iD https://orcid.org/0000-0003-30569065

Celia Oreja-Guevara (iD https://orcid.org/0000-00029221-5716

Heinz Wiendl (DD https://orcid.org/0000-0003-43103432

Tjalf Ziemssen (ID https://orcid.org/0000-0001-87998202

\section{Clinical Trial Information}

CAMMS223 [NCT00050778]; https://clinicaltrials. gov/ct2/show/NCT00050778

CARE-MS I [NCT00530348]; https://clinicaltrials. gov/ct2/show/NCT00530348

CARE-MS II [NCT00548405]; https://clinicaltrials. gov/ct2/show/NCT00548405

CARE-MS extension [NCT00930553]; https://www. clinicaltrials.gov/ct2/show/NCT00930553

TOPAZ [NCT02255656]; https://clinicaltrials.gov/ ct2/show/NCT02255656

\section{References}

1. Sanofi Genzyme. LEMTRADA (alemtuzumab): Summary of product characteristics, https://www. medicines.org.uk/emc/product/5409/smpc (2020, accessed 10 March 2020).

2. Cohen JA, Coles AJ, Arnold DL, et al. Alemtuzumab versus interferon beta 1a as first-line treatment for patients with relapsing-remitting multiple sclerosis: A randomised controlled phase 3 trial. Lancet 2012; 380: 1819-1828.

3. Coles AJ, Arnold DL, Bass AD, et al. Efficacy and safety of alemtuzumab over 6 years: Final results of the 4-year CARE-MS extension trial. Ther $A d v$ Neurol Disord 2021; 14: 982134.

4. Coles AJ, Compston DA, Selmaj KW, et al. Alemtuzumab vs. interferon beta-1a in early multiple sclerosis. N Engl J Med 2008; 359: 1786-1801.

5. Coles AJ, Twyman CL, Arnold DL, et al. Alemtuzumab for patients with relapsing multiple sclerosis after disease-modifying therapy: A randomised controlled phase 3 trial. Lancet 2012; 380: $1829-1839$

6. Ziemssen T, Bass AD, Berkovich R, et al. Efficacy and safety of alemtuzumab through 9 years of follow-up in patients with highly active disease: Post hoc analysis of CARE-MS I and II patients in the TOPAZ extension study. CNS Drugs 2020; 34(9): 973-988.

7. Genzyme Corporation. LEMTRADA REMS (risk evaluation and mitigation strategy), https://www. lemtradarems.com/ (accessed 13 May 2020).

8. National Cancer Institute. Surveillance, epidemiology, and end results program, https://seer. cancer.gov/statfacts/html/all.html (accessed 13 May 2020).

9. Kochanek KD, Murphy SL, Xu J, et al. Deaths: Final data for 2017. Natl Vital Stat Rep 2019; 68(9): 1-77.

10. Senior PA, Arnold DL, Cohen JA, et al. Incidence and timing of thyroid adverse events in patients with RRMS treated with alemtuzumab through 5 years of the CARE-MS studies. Neurology 2016; 86: P2086.
Visit SAGE journals online journals.sagepub.com/ home/msj

@SAGEjournals 